\title{
Multi-pulse scheme for laser-guided electrical breakdown of air
}

Pavel Polynkin

Citation: Appl. Phys. Lett. 111, 161102 (2017);

View online: https://doi.org/10.1063/1.4985265

View Table of Contents: http://aip.scitation.org/toc/apl/111/16

Published by the American Institute of Physics

\section{Articles you may be interested in}

Significantly extended cutoff wavelength of very long-wave infrared detectors based on $\ln \mathrm{As} / \mathrm{GaSb} / \mathrm{lnSb} / \mathrm{GaSb}$ superlattices

Applied Physics Letters 111, 161101 (2017); 10.1063/1.4998502

Diffraction-free, self-reconstructing Bessel beam generation using thermal nonlinear optical effect Applied Physics Letters 111, 161103 (2017); 10.1063/1.4997067

$\mathrm{PbSrS} / \mathrm{PbS}$ mid-infrared short-cavity edge-emitting laser on Si substrate Applied Physics Letters 111, 161104 (2017); 10.1063/1.4996259

Stretchable conductors by kirigami patterning of aramid-silver nanocomposites with zero conductance gradient Applied Physics Letters 111, 161901 (2017); 10.1063/1.5001094

Thermal conductivity of electron-irradiated graphene

Applied Physics Letters 111, 163101 (2017); 10.1063/1.4997772

Anisotropy-driven quantum capacitance in multi-layered black phosphorus Applied Physics Letters 111, 161902 (2017); 10.1063/1.4999380

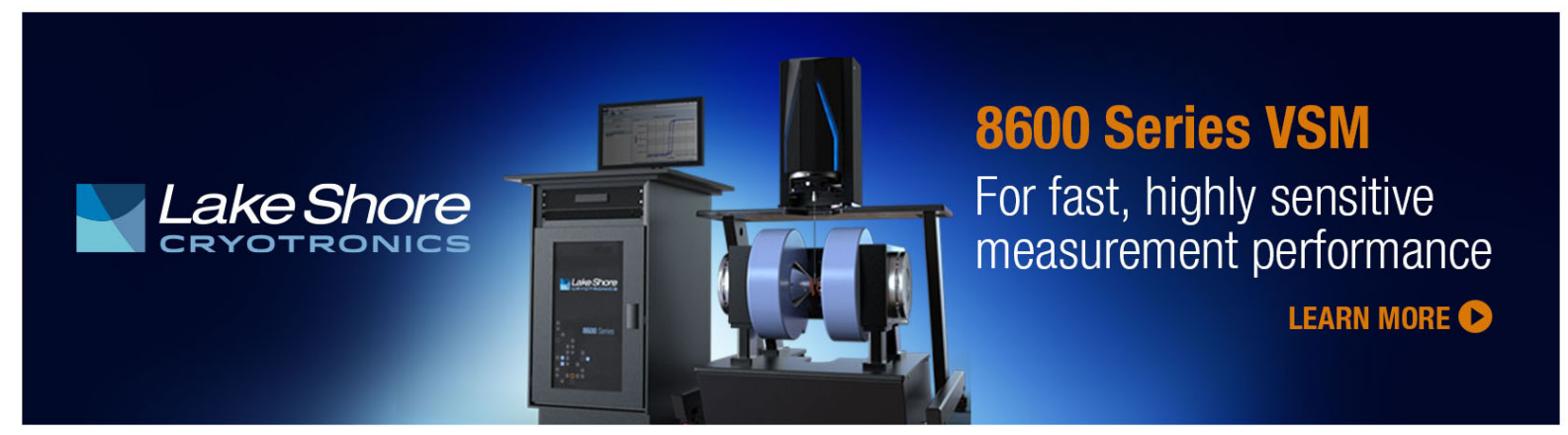




\title{
Multi-pulse scheme for laser-guided electrical breakdown of air
}

\author{
Pavel Polynkin ${ }^{\text {a) }}$ \\ College of Optical Sciences, University of Arizona, Tucson, Arizona 85721, USA
}

(Received 29 May 2017; accepted 1 October 2017; published online 16 October 2017)

\begin{abstract}
Channeling an extended electrical breakdown of air by a laser beam is a long-standing challenge in applied laser science. Virtually all previously reported experiments on discharge channeling by femtosecond laser beams relied on the application of a single laser pulse and have been conducted with discharge gaps of less than one meter, in which case the direct ohmic heating of the lasergenerated plasma by the applied DC electric field is the dominant channeling mechanism. We report a laboratory-scale demonstration of a channeling approach that makes use of concatenated plasma filaments produced by a sequence of multiple ultrashort laser pulses. Direct ohmic heating of the guiding channel is eliminated through the introduction of large temporal delays between the individual laser pulses in the pulse sequence. We propose an extension of this scheme to channeling kilometer-scale discharges, including natural lightning. Our proposed approach alleviates the fundamental range limitations inherent to the single-pulse schemes reported previously. It can channel discharges propagating in either direction and along curved paths. Published by AIP Publishing.

https://doi.org/10.1063/1.4985265
\end{abstract}

Lightning fascinated humans from the dawn of civilization. Scientific investigations of this natural phenomenon started during the times of Benjamin Franklin in the 18th century. These led to the invention of the lightning rod, ${ }^{1}$ which is a simple yet effective form of lightning protection widely used to this day. In the 20th century, the search for a more flexible solution explored the use of small rockets that leave behind a thin metal wire ${ }^{2}$ and, since about four decades ago, laser beams. ${ }^{3}$ Note that channeling air discharges with the length of several tens of meters, which is much shorter that the range of natural lightning and can also be of practical interest, e.g., for mine clearance.

In the 1970s-1990s, research on laser-guided electrical discharges focused on the utilization of plasma produced in the air by nanosecond laser pulses. Discharge guidance over the range of about $1 \mathrm{~m}$ has been demonstrated, ${ }^{4}$ but its length was fundamentally limited by the Rayleigh range of the focused laser beam. The additional limitation was associated with the spatial instability of air plasma produced by nanosecond lasers and its fragmentation into disconnected plasma bubbles. ${ }^{5} \mathrm{~A}$ single report on a presumably successful triggering of lightning in 1999 fell short of being convincing. ${ }^{6}$ The approach based on the use of nanosecond lasers has been deemed hopeless and abandoned.

Since the demonstration of ultrafast laser systems based on chirped-pulse amplification (CPA) and the subsequent demonstration of self-channeling propagation of intense ultrashort laser pulses in air, ${ }^{7}$ commonly referred to as laser filamentation, filament-based schemes for channeling the electrical breakdown of air have been actively investigated. ${ }^{8,9}$ The plasma channel produced through laser filamentation is not confined to the focal plane of an external focusing optic and appears to be ideally suitable for guiding a long-range atmospheric discharge. However, as we discuss below, the maximum range of guidance of air breakdown by a single

${ }^{\text {a)} E l e c t r o n i c ~ m a i l: ~ p p o l y n k i n @ o p t i c s . a r i z o n a . e d u ~}$ filament is still fundamentally limited by the distance on the order of ten meters, which is much shorter than what is necessary for channeling natural lightning.

The breakdown threshold electric field for air gaps shorter than few centimeters is $\sim 30 \mathrm{kV} / \mathrm{cm}$. For discharges longer than $\sim 10 \mathrm{~cm}$, including natural lightning, the breakdown is initiated by the discharge leader, which requires a lower DC electric field, between 5 and $10 \mathrm{kV} / \mathrm{cm}$, to propagate. ${ }^{10}$ The advancement of the leader relies on the heating of air at the tip of the leader, as well as on the enhancement of the local electric field due to the redistribution of space charges. Local heating and the subsequent reduction of the density of air result in the reduction of the breakdown threshold electric field in accordance with Paschen's law. ${ }^{11}$ In the natural (unguided) breakdown such as lightning, the heating is due to corona currents. The resulting propagation speed of the leader is on the order of $10^{6} \mathrm{~m} / \mathrm{s}$, which is two orders of magnitude slower than the speed of light. In the case of the breakdown guided by the femtosecond laser filament, there are two additional impulsive heating mechanisms: (i) Direct energy deposition by the laser field through strong-field ionization and impulsive rotational excitation of air molecules $^{9,12-17}$ and (ii) ohmic heating by the current driven by the applied DC electric field through the plasma filament connecting the electrodes. Both these heating mechanisms result in the formation of a low-density channel connecting the electrodes, which becomes a preferential path for the discharge to go through. The lifetime of this density hole is in the sub-millisecond range. It can be shown by a straightforward estimation that for short gaps, the second heating mechanism deposits significantly higher thermal energy into the air than the first mechanism that involves strong-field ionization and impulsive rotational excitation of molecules. This conclusion has been confirmed experimentally by observing the diffraction of a probe laser beam on the lowdensity channel produced by the laser filament with and without the high voltage present. ${ }^{9}$ It took tens of 
nanoseconds for the observable diffraction pattern to develop. That indicated that diffraction was on the air-density depression, not on the plasma, as the latter had a much shorter liifetime. Diffraction was only observed when the high voltage was turned on.

Plasma in the femtosecond laser filament has a lifetime on the order of a nanosecond. ${ }^{18}$ The corresponding instantaneous length of the plasma channel is less than $1 \mathrm{~m}$. Since the filament cannot electrically connect electrodes separated by the distance longer than its instantaneous length, the heating effect (ii), mentioned above, becomes inoperative, for gaps longer than $\sim 1 \mathrm{~m}$. This is why discharge guidance by the filament becomes much less effective when the distance between the electrodes is increased from below $\sim 1 \mathrm{~m}$, where both heating mechanisms contribute, to longer than $1 \mathrm{~m}$, where the heating has to rely on the strong-field mechanism alone. Yet, the majority of previously reported experiments on the guidance of electrical discharges of air by laser beams have been conducted with discharge gaps shorter than one meter, with few notable exceptions that reported the guidance of several-meter-long discharges. ${ }^{19-21}$ We will return to the discussion of the long-range discharge guidance below.

To demonstrate that an electrical discharge of air can be efficiently channeled by a laser filament without the ohmic heating mechanism present, we conduct the experiment schematically shown in Fig. 1. In this demonstration, we split the laser beam generated by an ultrafast Ti:Sapphire laser system, producing $15 \mathrm{~mJ}, 50 \mathrm{fs}$ laser pulses at the pulse repetition rate of 10 pulses per second, into three replica beams. We focus these three beams independently by separate $50 \mathrm{~cm}$ focal-length lenses and adjust the positions of the lenses so that the three formed plasma filaments are concatenated and bridge the gap between two disk electrodes charged to a DC voltage of $60 \mathrm{kV}$. The laser beams pass through holes drilled in the centers of the electrodes.

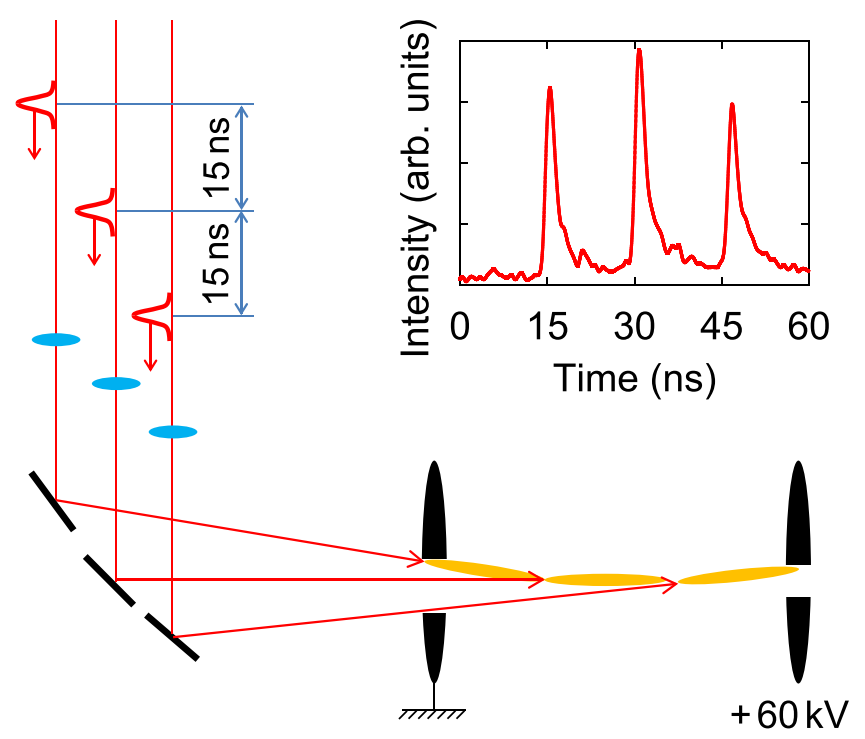

FIG. 1. Schematic diagram of the experiment on channeling air discharge by concatenated plasma filaments produced by three femtosecond laser pulses. Direct ohmic heating of air between the electrodes by the applied electric field is eliminated through the introduction of large temporal delays between the pulses. The inset shows the temporal waveform of the three-pulse sequence, measured using a photodetector in the far field.
The three pulses are temporally delayed with respect to each other by $15 \mathrm{~ns}$ using folded free-space delay lines. Such a delay is much longer than the nanosecond-scale plasma lifetime. The electron plasma generated by the first pulse decays, by several orders of magnitude, by the time the second pulse arrives to the inter-electrode gap and decays even further before the arrival of the third pulse. Even though some residual $\mathrm{O}_{2}^{-}$and $\mathrm{O}_{2}^{+}$ions remain for milliseconds after the passage of the laser pulses, ${ }^{22,23}$ such a heavy-ion plasma conducts very little current; the presence of these ions cannot appreciably affect the breakdown threshold electric field. Since there is no direct electrical connection between the high-voltage electrodes at any time, the channeling mechanism based on the ohmic heating is inoperative. In the particular implementation used here, the three-segment plasma wave and the resulting thermal excitation propagate against the direction of propagation of the laser beams, with the speed of about $3 \times 10^{6} \mathrm{~m} / \mathrm{s}$, which is on the same order as the speed of a natural discharge leader.

A photograph of the filament pattern produced by the sequence of the three delayed laser pulses is shown in Fig. 2(a). To make the faint fluorescence produced by the filaments visible in the photograph, the image has been integrated over 100 laser shots. The electrodes have been removed when this image was taken, in order to prevent the supercontinuum light, scattered by the right electrode, from overwhelming the image. A single-shot photograph of the $60 \mathrm{kV}$ discharge, guided by the filament sequence, is shown in Fig. 2(b). The $12 \mathrm{~cm}$-long discharge is reliably channeled by the sequence of the three laser pulses on every laser shot. The result is identical in the case when the delays between the pulses are reversed, corresponding to the thermal excitation wave copropagating with the laser beams.

The above multi-pulse scheme can be straightforwardly adapted to channeling discharges along curved paths. Two examples are shown in Fig. 3, where the three filaments and the corresponding guided discharges are configured in the dome (A, B) and zigzag (C, D) formations. In these
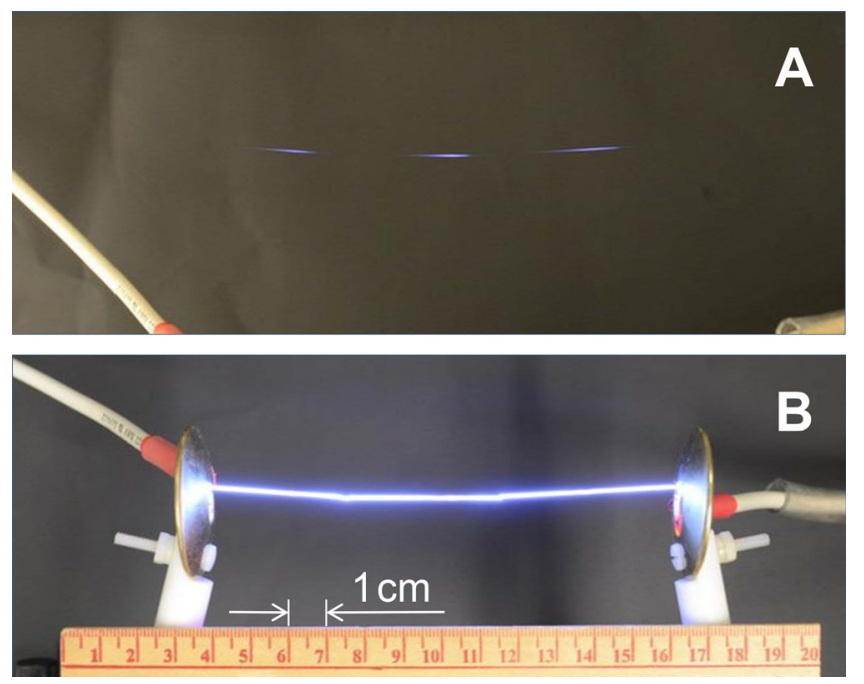

FIG. 2. (a) Photograph of fluorescence by three concatenated laser filaments, averaged over 100 laser shots. The laser beams propagate from left to right. (b) Single-shot photograph of the $12 \mathrm{~cm}$-long discharge channeled by the three-filament sequence. The right electrode is charged to $60 \mathrm{kV}$, and the left electrode is grounded. 

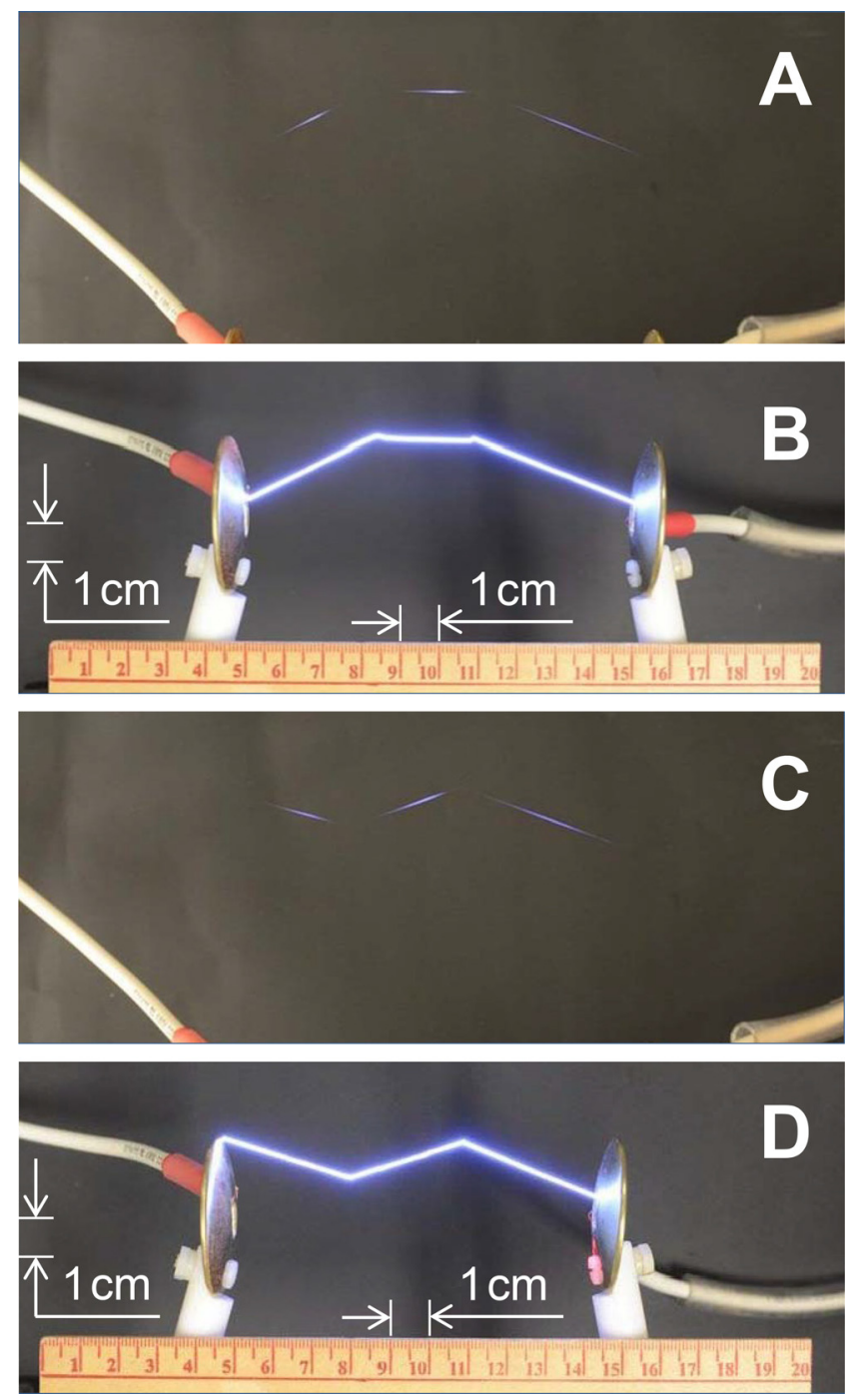

FIG. 3. Examples of curved discharges guided by timed sequences of three laser filaments in the dome and zigzag formations. (a), (c) Photographs of fluorescence by the filament plasmas, averaged over 100 laser shots. (b), (d) Single-shot images of the corresponding guided discharges.

examples, the deviation of guided $10 \mathrm{~cm}$-long discharges from a straight line is by about $2 \mathrm{~cm}$.

An alternative approach to channeling curved discharges is by using accelerating Airy beams. ${ }^{24}$ The transverse curvature of an Airy beam is limited to about one half of the overall beam size. Accordingly, the curvature of the discharge guided by a paraxial Airy beam, which has been reported in Ref. 24 , is only about $0.5 \mathrm{~mm}$, for a $5 \mathrm{~cm}$-long discharge, corresponding to the aspect ratio of $\sim 10^{-2}$. (Note that a much higher degree of transverse acceleration can be achieved with a non-paraxial Airy beam. ${ }^{25}$ However, non-paraxial Airy beams have only been demonstrated over sub-millimeter propagation distances, ${ }^{26}$ which is much shorter than what is practically interesting as far as the guided discharge is concerned.) In contrast, the multi-pulse approach discussed here is much more flexible. For example, the aspect ratio of the curved discharges shown in Fig. 3 is $\sim 0.2$. Also, note that the Airy beam-based channeling demonstration reported in Ref. 24 is an experiment with a short discharge gap, in which case the channeling is dominated by the direct ohmic heating of the laser-generated plasma by the applied DC electric field. This is not the case here.

For long gaps, channeling has to rely on the heat deposition by the strong-field mechanism alone. In that case, as has been shown in the experiment on the channeling of a $2.3 \mathrm{~m}$ long discharge, the guidance requires high laser-pulse energy on the order of $100 \mathrm{~mJ}$ per meter of guided discharge. ${ }^{19}$ The maximum guidance range is still limited, but now by the total integrated (not instantaneous) length of the plasma filament, which is on the order of several meters. Simply increasing the laser-pulse energy does not result in the significant extension of the filament length but causes transverse beam breakup into multiple short-range filaments that die out and reappear along the propagation direction. In principle, the filament range and continuity can be extended by beam shaping, ${ }^{27}$ beam combination, ${ }^{28}$ or the concatenation of several filaments produced through the beam focusing with a segmented lens. ${ }^{29}$ However, these approaches have their own range limitations associated with air dispersion and the wavefront flatness of the incident laser beam.

All guidance approaches investigated previously used lasers operating at low pulse repetition rates of up to $1 \mathrm{kHz} .{ }^{30}$ Accordingly, previous investigations relied on a single femtosecond laser pulse, a timed sequence of several femtosecond pulses, ${ }^{31}$ or a combination of a single femtosecond and a single nanosecond laser pulse. ${ }^{22,32,33}$ The limitations discussed above preclude single- or few-pulse schemes from being applicable to channeling atmospheric discharges with the length beyond $\sim 10 \mathrm{~m}$. Recent advances in the thindisk ultrafast laser technology had resulted in the development of CPA systems with multi-mJ pulse energies and MHz-scale pulse repetition rates. ${ }^{34}$ This technological progress, together with the recent increased awareness of thermal effects that accompany ultrafast laser filamentation in air, ${ }^{12-17}$ has inspired the multi-pulse channeling scheme that we discuss next.

The approach to the channeling of natural lightning that we propose is schematically shown in Fig. 4. Here, a train of multiple $\left(\sim 10^{3}\right)$ chirped ultrashort laser pulses creates a sweeping wave of thermal excitation of air. The pulse chirp varies from one pulse to the next at such a rate that the individual pulses are compressed by the natural dispersion of air at different distances, where these pulses undergo self-focusing collapse $^{35}$ and produce few-meters-long, concatenated plasma filaments. The direction of propagation and the speed of the thus created wave of thermal excitation of air can be adjusted to match the direction of propagation and the speed of the natural lightning leader. Similar schemes have been previously proposed for pumping backward-propagating air lasing ${ }^{36}$ and for the in-flight steering of supersonic projectiles. ${ }^{37}$

Channeling discharge in this scheme is predominantly through the deposition of thermal energy in the air. The resulting low-density path spans the entire distance between the thunderstorm cloud and the ground. An additional contribution to the channeling is through seeding the electron avalanche at the tip of the propagating discharge leader by the filament plasma. Through varying the rate of the pulse chirp, this scheme can be implemented to match the speed of either forward or backward-propagating leaders. This is an important flexibility since both upward and downward-propagating 


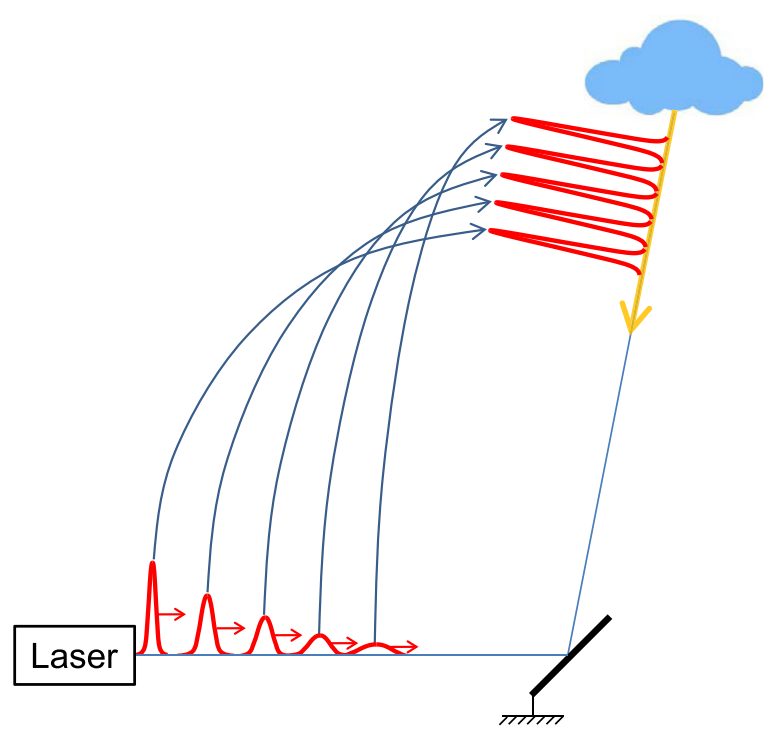

FIG. 4. Schematic diagram of the multi-pulse scheme for channeling lightning. The speed of the thermal excitation wave produced by a sequence of chirped laser pulses matches the speed of the natural lightning leader. The required time-varying pulse chirp can be straightforwardly produced by translating the mechanical delay stage in the pulse compressor of the CPA laser system.

leaders occur in thunderstorms, depending on the charge distribution in a particular thundercloud. In the case of channeling a forward-propagating leader, the propagation of a particular pulse in the pulse sequence will be affected by the density depression left behind by the preceding pulses. This influence, however, can only transversely shift the filament by the distance on the order of the transverse size of the density hole, which is negligible compared to the scale of lightning.

Note that our scheme is not about extending the subnanosecond-scale plasma lifetime in the femtosecond laser filament to several nanoseconds or even microseconds, as in the experiments reported earlier. ${ }^{22,31-33}$ This would be of little help for guiding a natural lightning strike, which is a process that involves the propagation of the lightning leader on a millisecond timescale. The key is to create a sweeping wave of air depression that spans the entire gap between the thundercloud and the ground in time on the order of a millisecond.

As an example of the implementation of the proposed channeling approach, consider lightning with the range of $1 \mathrm{~km}$. Assume that the laser system used for its channeling produces pulses at a center wavelength of $800 \mathrm{~nm}$ with the linewidth of $\Delta \lambda=30 \mathrm{~nm}$, corresponding to an approximately 30 fs FWHM duration of fully compressed Gaussian pulses. Assume that the pulse repetition rate is $1 \mathrm{MHz}$ and the energy per pulse is sufficient for self-focusing of the fully compressed pulses in air. The sweeping wave of thermal energy deposition will start at the thundercloud $1 \mathrm{~km}$ away from the ground and propagate downwards at the speed matching that of the natural lightning leader $\sim 10^{6} \mathrm{~m} / \mathrm{s}$. The typical variation of the pulse chirp with the detuning of the folded delay line in the pulse-compressor stage of an ultrafast CPA laser system is $\sim 1 \mathrm{ps} / \mathrm{mm}$. Using the value of the group-velocity dispersion of air $\left[-6 \times 10^{-2} \mathrm{ps} / \mathrm{nm} \cdot \mathrm{km}\right.$ (Ref. $38)]$, it can be straightforwardly estimated that the required speed of translation and the total travel range of the delay line are about $2 \mathrm{~m} / \mathrm{s}$ and $2 \mathrm{~mm}$, respectively. Such a mechanical performance is well within the capabilities of high-speed mechanical translation stages.

The resulting kilometer-long low-density guide will be produced by about one thousand linked filaments, separated from each other by a distance of about one meter. The sweeping excitation from the cloud to the ground (or vice versa) will occur within about one millisecond. Scanning the filament position by the pulse chirp and air dispersion can be aided by time-varying focusing of the laser beam. ${ }^{39}$ Channeling long-range curved discharges using this scheme would require two or more time-synchronized high-repetition-rate laser systems. Note that various practical issues relevant to our proposal need to be investigated, including the degree of control over the longitudinal placement of filaments that the sweeping pulse chirp allows and the influence of atmospheric turbulence.

In conclusion, we have experimentally shown that a series of concatenated laser filaments can channel a laboratory-scale electrical discharge of air in the case when the direct heating of the air channel between the electrodes by the applied electric field is not operative. The multipulse approach is shown to be suitable for guiding discharges propagating in either direction and along curved paths. We have proposed a scheme for the guidance of extended air discharges, including natural lightning, which is based on the application of multiple chirped femtosecond laser pulses. The required time-varying pulse chirp can be straightforwardly implemented by translating the delay stage in the pulse compressor of the CPA laser system. This approach alleviates the fundamental range limitations inherent to the single-pulse schemes suggested previously.

This material is based upon work supported by the U.S. Air Force Office of Scientific Research under MURI Award No. FA9550-16-1-0013.

${ }^{1}$ I. B. Cohen, Proc. Am. Philos. Soc. 96, 331 (1952).

${ }^{2}$ M. M. Newman, J. R. Stahmann, J. D. Robb, E. A. Lewis, S. G. Martin, and S. V. Zinn, J. Geophys. Res. 72, 4761, https://doi.org/10.1029/ JZ072i018p04761 (1967).

${ }^{3}$ L. M. Ball, Appl. Opt. 13, 2292 (1974).

${ }^{4}$ D. W. Koopman and T. D. Wilkerson, J. Appl. Phys. 42, 1883 (1971).

${ }^{5}$ M. Miki, T. Shindo, and Y. Aihara, J. Phys. D: Appl. Phys. 29, 1984 (1996).

${ }^{6}$ S. Uchida, Y. Shimada, H. Yasuda, S. Motokoshi, C. Yamanaka, T. Yamanaka, Z. Kawasaki, and K. Tsubakimoto, J. Opt. Technol. 66, 199 (1999).

${ }^{7}$ A. Braun, G. Korn, X. Liu, D. Du, J. Squier, and G. Mourou, Opt. Lett. 20, 73 (1995).

${ }^{8}$ X. M. Zhao, J.-C. Diels, C. Y. Wang, and J. M. Elizondo, IEEE J. Quantum Electron. 31, 599 (1995).

${ }^{9}$ S. Tzortzakis, B. Prade, M. Franco, and A. Mysyrowicz, Phys. Rev. E 64, 057401 (2001).

${ }^{10}$ V. Mazur, Principles of Lightning Physics (IOP Publishing, Bristol, UK, 2016).

${ }^{11}$ M. A. Lieberman and A. J. Lichtenberg, Principles of Plasma Discharges and Materials Processing, 2nd ed. (Wiley-Interscience, 2005).

${ }^{12}$ Y.-H. Cheng, J. K. Wahlstrand, N. Jhajj, and H. M. Milchberg, Opt. Express 21, 4740 (2013).

${ }^{13}$ N. Jhajj, E. W. Rosenthal, R. Birnbaum, J. K. Wahlstrand, and H. M. Milchberg, Phys. Rev. X 4, 011027 (2014).

${ }^{14}$ J. K. Wahlstrand, N. Jhajj, E. W. Rosenthal, S. Zahedpour, and H. M. Milchberg, Opt. Lett. 39, 1290 (2014). 
${ }^{15}$ E. W. Rosenthal, J. P. Palastro, N. Jhajj, S. Zahedpour, J. K. Wahlstrand, and H. M. Milchberg, J. Phys. B: At. Mol. Opt. Phys. 48, 094011 (2015).

${ }^{16}$ G. Point, C. Milian, A. Couairon, A. Mysyrowicz, and A. Houard, J. Phys. B: At. Mol. Opt. Phys. 48, 094009 (2015).

${ }^{17}$ O. Lahav, L. Levi, I. Orr, R. A. Nemirovsky, J. Nemirovsky, I. Kaminer, M. Segev, and O. Cohen, Phys. Rev. A 90, 021801(R) (2014).

${ }^{18}$ S. Tzortzakis, B. Prade, M. Franco, and A. Mysyrowicz, Opt. Commun. 181, 123 (2000).

${ }^{19}$ D. Comtois, C. Y. Chien, A. Desparois, F. Genin, G. Jarry, T. W. Johnston, J.-C. Kieffer, B. La Fontaine, F. Martin, R. Mawassi et al., Appl. Phys. Lett. 76, 819 (2000).

${ }^{20}$ M. Rodrigues, R. Sauerbrey, H. Wille, L. Wöste, T. Fujii, A.-B. André, A. Mysyrowicz, L. Klingbeil, K. Rethmeier, W. Kalkner et al., Opt. Lett. 27, 772 (2002).

${ }^{21}$ R. Ackermann, G. Méchain, G. Méjean, R. Bourayou, M. Rodriguez, K. Stelmaszczyk, J. Kasparian, J. Yu, E. Salmon, S. Tzortzakis et al., Appl. Phys. B 82, 561 (2006).

${ }^{22}$ B. Zhou, S. Akturk, B. Prade, Y.-B. Andre, A. Houard, Y. Liu, M. Franco, C. D'Amico, E. Salmon, Z.-Q. Hao, N. Lascoux, and A. Mysyrowicz, Opt. Express 17, 11450 (2009).

${ }^{23}$ P. Polynkin, Appl. Phys. Lett. 101, 164102 (2012).

${ }^{24}$ M. Clerici, Y. Hu, P. Lassonde, C. Milian, A. Couairon, D. N. Christodoulides, Z. Chen, L. Razzari, F. Vidal, F. Legare, D. Faccio, and R. Morandotti, Sci. Adv. 1, e1400111 (2015).

${ }^{25}$ A. Novitsky and D. Novitsky, Opt. Lett. 34, 3430 (2009).
${ }^{26}$ F. Courvoisier, A. Mathis, L. Froehly, R. Giust, L. Furfaro, P. Lacourt, M. Jacquot, and J. Dudley, Opt. Lett. 37, 1736 (2012).

${ }^{27}$ P. Polynkin, M. Kolesik, and J. Moloney, Opt. Express 17, 575 (2009).

${ }^{28}$ M. Scheller, M. Mills, M.-A. Miri, W. Cheng, J. Moloney, M. Kolesik, P. Polynkin, and D. Christodoulides, Nat. Photonics 8, 297 (2014).

${ }^{29}$ J. Papeer, R. Bruch, E. Dekel, O. Pollak, M. Botton, Z. Henis, and A. Zigler, Appl. Phys. Lett. 107, 124102 (2015).

${ }^{30}$ A. Houard, V. Jukna, G. Point, Y.-B. André, S. Klingebiel, M. Schultze, K. Michel, T. Metzger, and A. Mysyrowicz, Opt. Express 24, 7437 (2016).

${ }^{31}$ Z. Zhang, X. Lu, W.-X. Liang, Z.-Q. Hao, M.-L. Zhou, Z.-H. Wang, X. Liu, and J. Zhang, Opt. Express 17, 3461 (2009).

${ }^{32}$ M. Scheller, N. Born, W. Cheng, and P. Polynkin, Optica 1, 125 (2014).

${ }^{33}$ J. Papeer, M. Botton, D. Gordon, P. Sprangle, A. Zigler, and Z. Henis, New J. Phys. 16, 123046 (2014).

${ }^{34}$ K. Michel, S. Klingebiel, M. Schultze, C. Y. Tesseit, R. Bessing, M. Hafner, S. Prinz, D. Sutter, and T. Mertzger, Proc. SPIE 9726, 972614 (2016).

${ }^{35}$ P. Sprangle, J. R. Peñano, and B. Hafizi, Phys. Rev. E 66, 046418 (2002).

${ }^{36}$ P. R. Hemmer, R. B. Miles, P. Polynkin, T. Siebert, A. V. Sokolov, P. Sprangle, and M. O. Scully, Proc. Nat. Acad. Sci. U.S.A. 108, 3130 (2011).

${ }^{37}$ L. Johnson and P. Sprangle, J. Appl. Phys. 118, 123301 (2015).

${ }^{38}$ E. R. Peck and K. Reeder, J. Opt. Soc. Am. 62, 958 (1972).

${ }^{39}$ S. Eisenmann, E. Louzon, Y. Katzir, T. Palchan, A. Zigler, Y. Sivan, and G. Fibich, Opt. Express 15, 2779 (2007). 